\title{
Experience of Trained Primary Health Care Workers in Mental Health Service Delivery across Ogun State Nigeria
}

\author{
Adebowale Timothy ${ }^{1}$, Onofa lucky Umukoro ${ }^{*}$, Ighoroje Maroh ${ }^{1}$, Richard Gater ${ }^{2}$, Ogunwale \\ Adegboyega ${ }^{1}$, Okewole Niran ${ }^{1}$, Ogundele Adefolakemi ${ }^{1}$, Olarinde Samuel ${ }^{1}$, Olaitan Funmilayo ${ }^{1}$, \\ Olopade Modupe ${ }^{1}$ and Ogunyomi Karmodeen ${ }^{1}$
}

${ }^{1}$ Department of Neuropsychiatric, Neuropsychiatric Hospital, Aro, Ogun State, Nigeria

${ }^{2}$ Lancashire Care NHS Trust, Preston, United Kingdom

Corresponding author: Dr. Onofa Lucky Umukoro, MBBS, M.SC (Epidemiology), FWACP, Chief Consultant Psychiatrist \& WHO Global Mental Health Scholar, Department of Neuropsychiatric, Neuropsychiatric Hospital, Aro, Ogun State, Nigeria, Tel: +2348033336875; E-mail: onolucky@yahoo.com

Received date: March 1, 2017; Accepted date: March 28, 2017; Published date: April 4, 2017

Copyright: (c) 2017 Timothy A, et al. This is an open-access article distributed under the terms of the Creative Commons Attribution License, which permits unrestricted use, distribution, and reproduction in any medium, provided the original author and source are credited.

Citation: Adebowale T, Onofa LU, Ighoroje M, Gater R, Ogunwale A, et al. Experience of Trained Primary Health Care Workers in Mental Health Service Delievery Across Ogun State Nigeria. Clin Psychiatry 2017, 3:1.

\section{Abstract}

Background: In developing countries with shortage of mental health professionals, paramedics are trained in the delivery of mental health services. Knowledge of the experience of the trained health workers is useful in evaluating output of service and restructuring training package. This study was undertaken to determine the experience of trained primary health care (PHC) workers in mental health service delivery across Ogun State South west Nigeria.

Methods: Aro Primary Care Mental Health Program (APCMHP) was developed for the training of $80 \mathrm{PHC}$ workers across the state using adapted mhGAP intervention guide to assess and treat/ refer five priority conditions: Psychosis, Depression, Epilepsy, Alcohol \& Substance Abuse and Other Significant Emotional Complaint (OSEC). Data was collected on their experience 12 months after commencement of service delivery. Descriptive statistics were used and appropriate ethical approval was obtained.

Results: Out of the 80 PHC workers, attrition of 25 (31.3\%) was recorded in 12 months period. 54 valid questionnaires were analyzed. There were 52 (96.3\%) females and $90.7 \%$ were nurses. The mean (SD) age was 42.4 (16.3) years. A total of 473 patients were seen with diagnostic breakdown as follows: Psychosis (45.9\%), Epilepsy (38.3\%), Depression $(10.1 \%)$, OSEC (3.2\%) and Alcohol \& Substance Abuse $(2.5 \%)$. Diagnostic and treatment difficulty was reported for the following: Psychosis (23.1\%), Depression (22.0\%), Epilepsy (3.7\%), OSEC (52.2 \%) and Alcohol \& Substance Abuse (57.1\%). Personal fulfillment in mental service delivery was reported by $88.5 \%$, while $94.3 \%$ reported that the program was helpful to the community. Good attitude of clients and relatives towards the program was identified by $86.8 \%$ of the PHC workers.

Conclusion: Our study revealed a high personal fulfillment of the trained PHC workers in mental health service delivery. There is need for re-training of the PHC workers towards effective service delivery.

Keywords: Primary Health Care Workers; Experience; Mental Health Services; Oguh State; Nigeria

\section{Introduction}

Mental, neurological and substance use disorders account for $14 \%$ of the global burden of disease [1]. Low and medium income countries (LIMC) account for about $75 \%$ of this burden [2]. Despite evidence demonstrating that therapeutically safe, effective and affordable treatment is available, the proportion of the mentally ill who receive optimal treatment is alarmingly low [3]. The treatment gap range from $35-50 \%$ in High Income Countries (HIC) and 75-90\% in LMIC [4]. The problem is further compounded by the gross shortage of human and financial resources especially in developing countries [5].

Nigeria, an LMIC, with an estimated population of about 180 million people, has a lifetime prevalence of mental disorders ranging from $12.1-20 \%[6,7]$. Studies in Nigeria have found that only around $10 \%$ of those with MNS disorders receive any treatment within the previous 12 months [2]. Mental health services in Nigeria are widely underfunded. There is no specific allocation for mental health care in the national health budget. Mental health services are largely provided through eight specialists psychiatric unevenly distributed across the nation [8]. In terms of human personnel, there are less than 180 psychiatrists in the country (around 1 per 1 million population), around 5 psychiatric nurses per 100,000 population and only very few other mental and neurological health professionals like clinical psychologists, social workers, and occupational therapists [9].

To tackle the challenge and assist LMICs, such as Nigeria, in their effort to scale up mental health services the World Health Organization (WHO) launched the mental health Gap Action 
Programme (mhGAP) in 2008 [10]. The programmed aimed at narrowing the treatment gap by providing evidence based interventions for the most burdensome MNS conditions (depression, psychosis, dementia, epilepsy, child and adolescent behavioral and developmental disorders, alcohol use disorders, drug use disorders, and suicide). To achieve a wider coverage of the programme, the WHO has strongly recommended the integration of mental health care into primary health care services [2]. Integration of mental health care into primary care enables the largest number of people to gain faster and easier access to mental health care services, and can reduce stigma, improve social integration and improve human resources for mental services $[11,12]$. The structure of the primary health care (PHC) system in Nigeria provides a good platform for the delivery of mental health services in the community [13].

In developing countries as Nigeria, with shortage of professionals, paramedics (nurses and community health workers) often deliver health services in primary health centers. Often these workers have little or no formal training in the delivery of mental health care. The process of integrating mental health care into primary health care requires training of $\mathrm{PHC}$ workers in detection, diagnosis, treatment and timely referral of individuals with mental disorders. The adequately trained PHC worker is well positioned to deliver mental health services in the local community [14]. This study was undertaken to determine the experiences of trained Primary Health Care workers in mental health service delivery across Ogun state, South-West, Nigeria. This knowledge is useful in evaluating output of service and restructuring the training package.

\section{Objectives}

The objectives of the study were to describe the sociodemographic profile of PHC workers in Ogun state, South-West, Nigeria, to determine the caseload of patients with mental illness seen over a 12-month period and to document the experience of the trained PHC workers in mental health service delivery.

\section{Materials and Methods}

Ogun State is located in the South-West of Nigeria with a total land area of 16,409.26 square kilometers and an estimated population of about 4 million [15]. The state comprises of 20 local government areas, grouped into four zones: Egba, Yewa, Remo and ljebu with five local government areas in each zone. There are more than 300 PHC facilities in Ogun State, with primary care manpower ranging from 4 to 20 at each facility [14]. In 2011, the Neuropsychiatric Hospital Aro, Nigeria (the only tertiary psychiatric facility in the state) embarked on primary care mental health services in selected primary health care centers across the state [16].

Four PHC workers were selected for mental health training from each of the 20 local government areas making a total of 80 PHC workers. Forty health centers were selected for mental health service delivery. Seventy (87.5\%) of the workers were nurses while $10(12.5 \%)$ were community health workers. The selection was based on the health workers' interest in delivery of mental health services in their practice.

Training materials were based on the WHO's mental health Gap Action Programme (mhGAP) intervention Guide (2008), which was adapted to suit local circumstances [10]. The mhGAP Document was developed by WHO in line with ICD categories. The scope of the training was to assess and treat five priority psychiatric disorders including Psychosis, Depression, Other Significant Emotional Complaints (OSEC), Epilepsy and Alcohol \& Substance abuse. The OSEC is the abbreviation for Other Significant Emotional Complaints and the disorders under OSEC are Anxiety disorders, Somatoform disorders, mild depression, post-traumatic stress disorders, acute stress reaction, adjustment disorders and medically unexplained symptoms. A preliminary survey during the pilot phase of the project found that the 5 conditions were prevalent in Ogun state.

A 3-day training course was developed to equip the 80 primary care workers with knowledge and skills to diagnose, treat and/or refer people with the five priority disorders. The planning and implementation of the training course was done in collaboration with the University of Manchester/Lancashire Care NHS Foundation Trust UK, under a British Council's Health-Link Scheme. The training was delivered by faculty members from Neuropsychiatric Hospital, Aro and Lancashire Care Trust using didactic and participatory methods including lectures, video demonstrations, role plays, exercises and discussions. Four, 2day training sessions were carried out in locations within the four socio-political zones of Ogun State with 20 PHC workers in each zone, following a one-day plenary introductory lecture on classification, causes and treatment approaches in mental disorders at the Neuropsychiatric hospital Aro Abeokuta. The training was conducted between September and October, 2011. Written support materials, including assessment flow charts, case record and follow-up sheets were developed for the primary care workers to guide their practice and keep record of their practice. Essential psychotropic drugs were provided at the selected health and a drug revolving fund set-up. The programme received wide support from the relevant stakeholders (traditional rulers, religious and community leaders, non-governmental organizations and officials of the state ministry of health). A framework was put in place to support and supervise the trained primary care worker. This is done through regular visits and telephone support by psychiatric nurse supervisors. Follow-ups and referral for psychiatric consultant review was also put in place to facilitate the sustainability of the project.

Twelve months after commencement of mental health service delivery in the PHC facilities, data was collected on the experiences (patients' caseload, difficulties in making a diagnosis and offering treatment, field experiences and service perception) of the trained primary health care worker. Data were collected using semi-structured questionnaire by the authors which contains two sections; A (Socio-demographic characteristics of the patients and section $B$ which elicits information on mental health conditions presenting at the health centers, psychotropic medications supply, use of flow chart, use of record sheets, problems with case diagnosing, case 
referral, follow up, helpfulness of the program, personal fulfillment in participating in the program and level of support towards the program. See Appendix 1 for details of the Questionnaire. Data were analyzed using SPSS version 20 [17]. Results were presented using descriptive statistics, frequencies and percentages. Ethical approval was granted by the Hospital Research Ethics Committee, and consent of the PHC workers was obtained for the study.

\section{Results}

Of the 80 trained PHC workers, 55 (68.8\%) continue to deliver mental health services in primary care 12 months after the commencement of the project. The Attrition rate was $31.2 \%$ i.e. 25 of the trained PHC workers resigned retired, or were transferred out of the designated primary health center. Fifty four (54) valid questionnaires were analyzed. The sociodemographic profile showed that the majority of the workers were females (96.3\%). The mean (SD) age was 42.4 (16.3) years. Over $90 \%$ of the workers were nurses (Table 1).

Table1 Socio-demographic characteristics of PHC workers N=54.

\begin{tabular}{|l|l|l|}
\hline Characteristics & Pale & Proportion (\%) \\
\hline \multirow{4}{*}{ Gender } & Female & 3.7 \\
\hline \multirow{4}{*}{ Education } & Diploma & 96.3 \\
\cline { 2 - 3 } & B. Sc & 27.8 \\
\cline { 2 - 3 } & Registered Nurse \& Midwife & 59.2 \\
\hline \multirow{4}{*}{ Marital Status } & Single & 7.4 \\
\cline { 2 - 3 } & Married & 88.9 \\
\cline { 2 - 3 } & Divorced/Widowed & 3.7 \\
\hline \multirow{3}{*}{ Marital Status } & Nurse & 90.7 \\
\cline { 2 - 3 } & $\begin{array}{l}\text { Community } \\
\text { Extension Worker }\end{array}$ & 9.3 \\
\hline
\end{tabular}

Four Hundred and seventy three patients were seen during the 12 months period of which 217 (45.9\%) had psychosis. This was closely followed by epilepsy (38.2\%), depression (10.1\%), OSEC (3.2\%) and alcohol/substance abuse (2.5\%) (Figure 1).

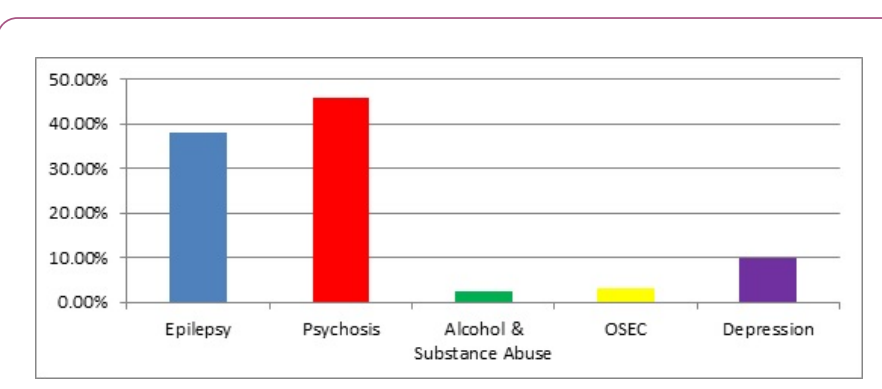

Figure 1 Caseload of patients seen in a 12-month period.

With regards to their experiences in making a diagnosis and carrying out treatment interventions, the workers reported that epilepsy was the easiest to diagnose and the least difficult to treat. Only $3.7 \%$ of the PHC workers expressed difficulties in diagnosing and treating epilepsy. Although about 1 of every 4 PHC workers expressed difficulty in diagnosing psychosis and depression, fewer workers had challenges offering treatment once diagnosis was made. More than half of the trained workers had difficulties diagnosing OSEC and offering treatment interventions. Offering treatment for alcohol and substance abuse posed the greatest difficulties (57.1\%) for the PHC workers (Table 2).

Table 2 Reported experience of the trained PHC workers $\mathrm{N}=54$.

\begin{tabular}{|l|l|l|}
\hline Diagnosis & $\begin{array}{l}\text { Difficulty in making } \\
\text { Diagnosis (\%) }\end{array}$ & $\begin{array}{l}\text { Diagnosis in Treatment } \\
\text { Intervention (\%) }\end{array}$ \\
\hline Psychosis & 23.1 & 13.5 \\
\hline Epilepsy & 3.7 & 3.7 \\
\hline Depression & 22 & 19.1 \\
\hline OSEC & 52.2 & 50 \\
\hline $\begin{array}{l}\text { Alcohol \& substance } \\
\text { abuse }\end{array}$ & 38.6 & 57.1 \\
\hline
\end{tabular}

The over-whelming majority of the trained workers reported a high service perception and positive field experiences. Seventyfive percent reported good support from their community, and a good public awareness of their services. Over $88 \%$ reported a high personal fulfillment and good support from other nontrained PHC workers, while $98 \%$ said they received good support from the Aro Supervisory team Table 3.

Table 3 On the field experience of trained PHC workers and service perception $\mathrm{N}=54$.

\begin{tabular}{|c|c|}
\hline Characteristics & Proportion (\%) \\
\hline High personal fulfillment of PHC workers & 88.5 \\
\hline Good support from other workers & 88.7 \\
\hline Good support from community & 75.5 \\
\hline Good support from Aro supervisory team & 98.1 \\
\hline Helpfulness of service to community & 94.3 \\
\hline Good attitude of service users \& relatives & 86.8 \\
\hline Good public awareness of service & 75.6 \\
\hline
\end{tabular}

\section{Discussion}

In this study, most of the PHC workers were females and nurses. These profiles are similar to findings from previous studies $[18,19]$. In low and medium income countries with shortage of material, financial and human resources, such as Nigeria, primary health care services are primarily provided by paramedics [12]. However, in more developed settings, as in the United Kingdom, qualified medical personnel's usually lead the primary mental health care team [12].

Majority of the health workers had had previous exposure to mental health courses during their training and had come in 
contact with mentally ill patients during their practice but most of the cases they saw then were advised and were sent back home. The choice to participate was based on voluntariness and interest in mental health service delivery.

In our previous publication on evaluation of mental training course for the primary health care workers involved in mental health service delivery, a pre and post test showed that mental health training course for PHC workers resulted in improved knowledge and expected mental health practice with greater effects on case management than case recognition [14].

A high attrition rate of $31.2 \%$ was reported in this study. This is quite common among mental health workers and has been previously documented in Nepal, India [20,21]. The high attrition rate thwarts training initiatives since the proportion of remaining staff dwindles over time and frustrates the development of services $[20,22]$.

To reduce the attrition of trained PHC workers, it is appropriate to train only primary care staffs who are interested in mental health matters and who are likely to remain in their posts for a long time. Tasks assigned should be limited, doable and take consideration of local circumstances and the worker's additional and competing responsibilities for treating physical disorders [22]. In addition to pre-service training, project coordinators should organize regular in-service training to reinforce and update knowledge and practice. Supervisors also need to be trained and retrained, so that they have a good understanding of their supervisory role and skills [23].

Four hundred and seventy three patients were diagnosed and treated for mental and neurological disorders during the 12 months. Similar to this finding, an extensive body of investigation has consistently reported that a significant number of patients seen in primary health care present with medically unexplained symptoms and comorbid mental illnesses [24-26]. A high proportion of the patients $(45.9 \%)$ in this study were diagnosed as having psychosis, while only $10 \%$ had a depressive disorder. This result is quite contrary to several other studies that reported mild-moderate depression, dysthymia, anxiety, somatoform and alcohol use disorders as the most common disorders in primary mental health care delivery [25,27-29].

The high rate of psychosis may be partly explained by the bridge in the pathway of presentation where acutely ill psychotic patients present early on to the formal health sector, rather than first presenting at an informal unorthodox setup. Prior to the commencement of the project the supervisory specialist nurses and trained PHC workers embarked on community enlightenment and mental health advocacy. This may have led to an increased awareness in the community of mental health services in the designated primary health care centers.

The report of a lower rate of depression among patients seen in this study may be explained culturally. Detecting mildmoderate depression in primary care poses enormous challenges since many patients may present with somatic symptomatology such as headaches and body pains. This often makes the use of western diagnostic classifications difficult [6]
In this study, $25 \%$ to over half of the trained PHC workers reported difficulties in diagnosing depression and Other Significant Emotional Complaints (OSEC). In addition, symptoms may cross diagnostic boundaries (such as mood, anxiety and somatoform disorders) [30].

Even-though the health workers received an interactive course in mental health, a significant number reported difficulties in diagnosing and offering treatment interventions. For example, more than half of the trained workers had difficulties diagnosing OSEC and offering treatment interventions for alcohol and substance abuse. Though many cases of depression are suitable for treatment, a systematic review noted that only half of all cases of depression in primary care setting are recognized [31].

Difficulties to diagnosing and treating mental disorders in primary care includes: knowledge and skill deficit, stigma, patient's somatization, denial and limited time per patient. One area of debate is that the current diagnostic criteria (International Classification of Diseases-ICD; Diagnostic Statistical Manual-DSM) were developed largely in psychiatric settings, and may not readily apply to primary care and other medical settings [32]. This is because symptomatic patients, with varying degrees of impairment, but who fails to meet the full diagnostic criteria for a particular mental disorder are frequently encountered in primary care and may be inappropriately diagnosed or treated. Increase public education, continuous training of the heath care workers and supervisors are needed to reduce these barriers [31].

The high perception of services and the positive field experiences (such as community utilization of mental health services) reported by the workers showed the feasibility of training primary health care workers in the identification, diagnosis and first line treatment of mental disorders. This training which was done via a three day interactive course in mental health, using specialist trainers, limited funding, an advocacy and supervisory support network resulted in an improved knowledge, attitude, practice, and transfer of skills.

Integrating mental health into primary health care remains the most viable option in reducing the burden of mental illness in the society. However, in Nigeria, this process is haphazard and nationally uncoordinated [33]. To enable a national coverage, it is recommended that the health ministries, departments and agencies intensify efforts to train primary care workers in mental health issues. This can be done through intersect oral partnerships in the context of the nation's general health or mental health policy.

\section{Limitation}

The choice to participate was voluntary and was also based on the interest of the PHC workers. However, the differential level of interest could confound some of the findings of this study. However, the purposeful selection of participants is required for the success of the program. 


\section{Conclusion}

Our study revealed a high personal fulfillment of the trained $\mathrm{PHC}$ workers in mental health service delivery. There is need for re-training of the PHC workers towards effective service delivery.

\section{References}

1. Whiteford HA, Ferrari AJ, Degenhardt L, Feigin V, Vos T (2015) The global burden of mental, neurological and substance use disorders: an analysis from the global burden of disease study 2010. PloS one 10: e0116820.

2. World Health Organization (2010) Mental Health Gap Action Programme: MhGAP Intervention Guide for Mental, Neurological and Substance Use Disorders in Non-specialized Health Settings 1 : 86-94.

3. Kohn R, Saxena S, Levav I, Saraceno B (2004) The treatment gap in mental health care. Bull World Health Organ 82: 858-866.

4. Funk M, Drew N, Knapp M (2012) Mental health, poverty and development. Journal of Public Mental Health 11: 166-185.

5. Scheffler RM (2011) Human resources for mental health: workforce shortages in low and middle income countries. Human Resources for Health Observer 8.

6. Gureje O, Lasebikan VO, Kola L, Makanjuola VA (2006) Lifetime and 12-month prevalence of mental disorders in the Nigerian Survey of Mental Health and Well-Being. Br J Psychiatry 188: 465-471.

7. Abdulmalik J, Fadahunsi W, Kola L, Nwefoh E, Minas $\mathrm{H}$, et al. (2014) The Mental Health Leadership and Advocacy Program (mhLAP): a pioneering response to the neglect of mental health in Anglophone West Africa. Int J Ment Health Syst 8:1.

8. World Health Organization (2010) WHO-AIMS: Mental Health Systems in Selected Low-and Middle-income Countries: a WHOAIMS Cross-national Analysis: World Health Organization.

9. Federal Ministry of Health Nigeria (2003) National Policy for Mental Health Services Delivery Nigeria: 1-22.

10. World Health Organization (2016) mhGAP intervention guide for mental, neurological and substance use disorders in nonspecialized health settings. Lancet.

11. Budosan B (2011) Mental health training of primary health care workers: case reports from Sri Lanka, Pakistan and Jordan. Intervention 9: 125-136.

12. World Health Organization (2008) Integrating mental health into primary care: a global perspective: World Health Organization.

13. Abiodun O (1995) Pathways to mental health care in Nigeria. Psychiatric services 46: 823-826.

14. Adebowale T, Onofa LU, Gater R, Akinhanmi A, Ogunlesi A, et al. (2014) Evaluation of a Mental Health Training Course for Primary Health Care Workers in Ogun State, South West, Nigeria. J Psychiatry 17: 5.

15. Commission NP (2006) Population and housing census of the Federal Republic of Nigeria. Priority tables.

16. Onofa LU (2013) Development and roll-out of primary care mental health services in Ogun state, Nigeria. Commonwealth Health Partnership: 97-99.
17. Green SB, Salkind NJ (2010) Using SPSS for Windows and Macintosh: Analyzing and understanding data: Prentice Hall Press.

18. Odejide O, Morakinyo J (2003) Mental health and primary care in Nigeria. World psychiatry : official journal of the World Psychiatric Association (WPA)2: 164.

19. Ekwueme O, Aghaji M (2006) The concept and attitudes of primary health care workers towards mental illness in rural Igbo land of Nigeria. Int J Med Health Development 11:125-129.

20. Abas M, Baingana F, Broadhead J, lacoponi E, Vanderpyl J (2003) Common mental disorders and primary health care: current practice in low-income countries. Harv Rev Psychiatry 11:166-173.

21. Ackland S (2002) Mental health services in primary care: the case of Nepal. World mental health casebook New York: Kluwer Academic/Plenum Publishers: 121-152.

22. Mohit A (2013) Mental health in Tehran in the context of the national mental health programme of Iran. Mental health in our future cities Maudsley Monograph 12: 217-238.

23. Jenkins R, Kiima D, Njenga F, Okonji M, Kingora J, et al. (2010) Integration of mental health into primary care in Kenya. World J Psychiatry (WPA) 9: 118-120.

24. Schulberg HC, Burns BJ (1988) Mental disorders in primary care: epidemiologic, diagnostic, and treatment research directions. General Hospital Psychiatry 10: 79-87.

25. Ansseau M, Dierick M, Buntinkx F, Cnockaert $P$, De Smedt J, et al. (2004) High prevalence of mental disorders in primary care. J Affect Disord 78: 49-55.

26. Cowen P, Harrison P, Burns T (2012) Shorter Oxford textbook of psychiatry: Oxford University Press.

27. Sartorius N, Üstün TB, Lecrubier Y, Wittchen HU (1996) Depression comorbid with anxiety: Results from the WHO study on "Psychological disorders in primary health care". The British journal of psychiatry 30: 38-43.

28. Ormel J, VonKorff M, Ustun TB, Pini S, Korten A, et al. (1994) Common mental disorders and disability across cultures: results from the WHO Collaborative Study on Psychological Problems in General Health Care. Jama 272: 1741-1748.

29. Kroenke K, Spitzer RL, Williams JB, Monahan PO, Löwe B (2007) Anxiety disorders in primary care: prevalence, impairment, comorbidity, and detection. Ann Intern Med 146: 317-325.

30. Semple D, Smyth R (2013) Oxford handbook of psychiatry: Oxford University Press.

31. Goldman LS, Nielsen NH, Champion HC, for the Council on Scientific Affairs AMA (1999) Awareness, Diagnosis, and Treatment of Depression. J Gen Intern Med 14: 569-580.

32. Wittchen HU, Höfler M, Meister W (2001) Prevalence and recognition of depressive syndromes in German primary care settings: poorly recognized and treated. Int Clin Psychopharmacol 16:121-135.

33. Odejide AO, Morakinyo JJ, Oshiname FO, Omigbodun O, Ajuwon A, et al. (2001) Integrating mental health into primary health care in Nigeria: management of depression in a local government (district) area as a paradigm. Seishin Shinkeigaku Zasshi 104: 802-809. 